\title{
Corneal Cross-Linking Procedure in Progressive Keratoconus Treatment
}

\author{
Arleta Waszczykowska* \\ Department of Ophthalmology and Vision Rehabilitation, Poland
}

Received: February 01, 2018; Published: February 12, 2018

*Corresponding author: Arleta Waszczykowska, Department of Ophthalmology and Vision Rehabilitation, Medical University of Lodz, Zeromskiego 113, 90-549 Lodz, Poland, Tel: 48 426393634; Email: arletawaszczykowska@interia.pl

\begin{abstract}
Keratoconusis a progressiveeye disease, whichdiscloses in adolescence and usuallystabilizes in thefourthorfifthdecadeoflife. Theprogressivethinningofcorneal stroma in which the cornea has a conical shapeleads to high irregular a stigmatism and consequently to a significantvisualacuitydecrease. Anymethod to curethediseasehas beendeveloped up to thisday. Corneal collagen cross-linking (CXL) is the first treatment that halts the progression of keratoconus. Cross-linkingconducted in the early stages of keratoconus allows to stop cornealchangesduring early grade, easier to opticalcorrection. From the times of "Dresden Protocol" until now, multiple variations of the standard procedure technique have been introduced and consistent improvement is still being searched for.Not onlydoesthe study presenttheclassicalmethodof CXL, acceleratedand transepithelial CXL modifications, but thelatestresearchabouttheireffectiveness as well.
\end{abstract}

Keywords: Corneal cross-linking; CXLl; Accelerated cross-linking; High irradiance; Transepithelial cross-linking; Keratoconus

\section{Introduction}

\section{A literature review}

Cross-linking (X-linking, CXL) is a surgical procedure designed to strengthen the corneal structure and stop the progression of keratoconus. Cross-linking method was applied for the first time in Technical University in Dresden (Germany) in 1998 [1]. Efficacy and safety of the method was later confirmed in multiple clinical studies conducted worldwide [2,3]. Range of indications for CXL procedure is currently widened to include, e.g. treatment of post-operative corneal ectasia, bullous keratopathy, lattice corneal dystrophy, infectious corneal ulceration (Photo Activated Chromophore for Keratitis Cross-linking, PACK-CXL). The procedure is also sometimes combined with refractive surgery of the cornea (CXLPlus) [4]. Also some attempts were made to apply CXL procedure as supplementary to orthokeratology, keratoplasty or for the treatment leaking cystic blebs after trabeculectomy [5,6]. The most recent reports indicate that scleral cross linking with riboflavin and ultraviolet-A radiation effectively prevents axial myopia in a rabbit model [7].

Standard CXL methodology involves removal of corneal epithelium (Epithelium-Off) to improve penetration of riboflavin (vitamin B2), as epithelium provides a natural diffusion barrier for it. Repeated applications (for approx. 30 minutes) of $0.1 \%$ riboflavin solution in $20 \%$ dextran enable high riboflavin concentration in the corneal stroma to be achieved. Appropriate saturation of the corneal stroma with riboflavin has been proven to multiply many times absorption of radiation by anterior corneal layers, which prevents penetration of UVA into deeper eye structures [2]. The cornea is exposed to ultraviolet radiation at wavelength of $365 \mathrm{~nm}$ (UVA) and power of $3 \mathrm{~mW} / \mathrm{cm}^{2}$ for subsequent 30 minutes, with concomitant application of riboflavin (Figure1).

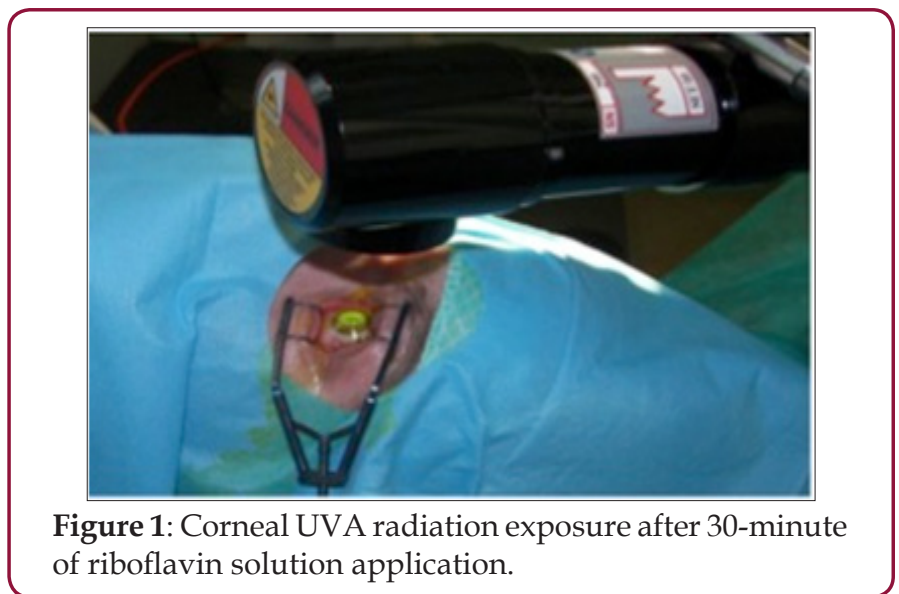

Duration of the procedure is a drawback of CXL method. Standard procedure lasts 1 hour, which is burdensome for the patients and significantly limits number of procedures that may be performed. Moreover, epithelium removal requires relatively prolonged process of epithelium restoration and is frequently 
associated with eye pain and necessity of wearing protective contact lenses $[1,8]$. Numerous modifications of standard CXL technique was implement until now, some of them aimed at the protection of corneal epithelium, while others - with tendency to reduce the procedure duration.

\section{Various Aspect of Cxl Methodology}

The procedure is performed in patients with progressive morphologic changes in the course of low-stage of keratoconus (stage 1, 2 or sometimes 3 according to Amsler-Krumeich's classification). Progression of keratoconus is defined as fulfilling of at least one of the following criteria: increase in Kmax of 1 diopter (D) in 1 year, or a change in either myopia and/orastigmatism $\geq 3$ D in 6 months, a mean central K-readingchange $\geq 1.5$ D observed in threeconsecutivetopographies in 6 months, or a mean central cornealthicknessdecrease $\geq 5 \%$ in threeconsecutivetomographies in the previous 6 months [9]. Safety of the CXL procedure, apart from the UVA dose applied, also depends on the corneal thickness, which should not be lower than $400 \mu \mathrm{m}$. Such a corneal thickness is known to prevent successfully damage to endothelial cells during UV irradiation. Effect of the procedure has been shown to be limited to 200-350 $\mu \mathrm{m}$ of corneal thickness (Figure 2).

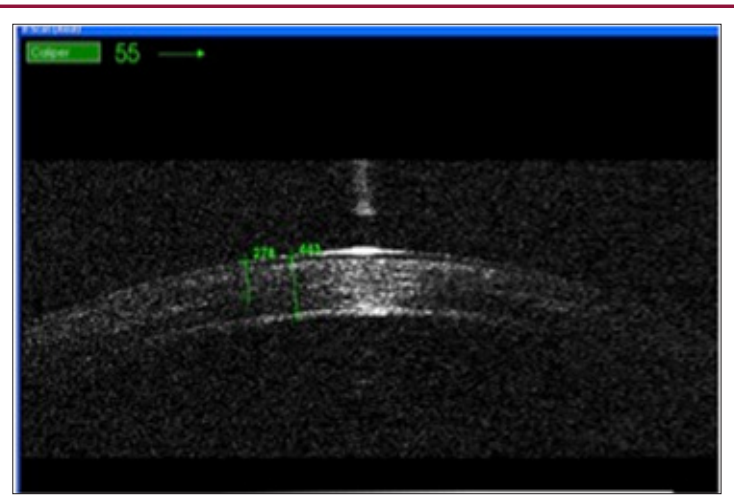

Figure 2: Optical Coherence Tomography image showing demarcation line at $278 \mu \mathrm{m}, 24$ months after accelerated CXL.

However, it should be borne in mind that during CXL procedure corneal thickness is reduced, by $19 \%$ on the average, due to dehydration $[10,11]$. The process is also facilitated by thinning of

Table 1: Complications after CXL procedure. corneal stroma following epithelium removal. Reduction of the corneal thickness during the CXL procedure is known to occur in two stages. During the first stage, involving a 30-minute period of riboflavin administration, corneal thinning may even amount to $15 \%$. The patient is recommended to keep their eyes closed and open them only for the moment of the drop instillation. Such an approach allows changes detected in pachymetry to be reduced and may even result in insignificant improvement (approx. 1\%) of corneal thickness [12]. The other and more intense stage of corneal thinning involves the UVA irradiation phase[10]. Relatively high rate of CXL procedure complications (7.6\%) enforces rigorous qualification of patients to the procedure[5].

The following exclusion criteria for cross-linking procedure are mentioned:

a. Pre-operative keratometry results $\leq 58 \mathrm{D}$.

High keratometry results are positively correlated with the risk of post-operative keratoconus progression and permanent stromal haze. Exclusion of such patients reduces general risk of procedure complications to $2.8 \%$.

b. Age $>35$ years. Risk of complications in young patients is $1.04 \%$.

c. Corneal thickness following epithelial abrasion $<400 \mu \mathrm{m}$.

Corneal thickness following lower than $400 \mu \mathrm{m}$ significantly increases the risk of endothelial cells damage.

d. Visual acuity 20/25 or better.

There is a risk of lower acuity after the procedure. Lack of any effect of CXL procedure is noted in patients with high pre-operative keratometry results, as well as in patients with neurodermitis and pregnant women, likely due to hormonal disturbances [5]. Question of efficacy of CXL procedure is raised in numerous publications, while there is much less information concerning actual and possible complications. Possible complications following CXL procedure are presented in Table 1 [5,13-16].It has been proven that CXL procedure is associated with keratocyte apoptosis, atrophy of nerve plexuses and oedema of anterior layers of corneal stroma. Average time for complete epithelial re-epithelisation is 5 days.

\begin{tabular}{|c|c|}
\hline \multicolumn{2}{|c|}{ Complications after CXL procedure } \\
\hline Early & Late \\
\hline Chronic pain & Sterile corneal infiltrates \\
\hline Photophobia & Infectiouskeratitis (bacterial, viral, Acanthamoeba) \\
\hline Fixed excessive corneal sensitivity to temperature changes & Delayed healing of the epithelium \\
\hline Reduction of visual acuity & Recurrent keratitis \\
\hline The "glare" phenomenon & Focal, transient or permanent corneal opacity \\
\hline Corneal edema & Permanent scars in corneal stroma \\
\hline Dense mildstromal opacity (Figure 3) & Corneal degeneration (the demarcation line within the area of corneal abrasion) \\
\hline Ciliary hyperemia & Endotheliopathy with corneal edema \\
\hline Inflammatorycells in aqueous humor & Keratouveitis \\
\hline
\end{tabular}




\author{
Herpetickeratitis \\ Transientanisocoria
}

Infectious crystalline keratopathy

Reactivation of herpes simplex

Perforation and corneal melt
Process of restoration of subepithelial nerve plexus starts approx. 1 month after the procedure, while keratocyte repopulation 3-6 months after CXL procedure. New collagen fibres rebuilt in these processes are thicker, more rigid and more resistant to extracellular matrix enzymes [5]. According to abundant literature, rate of the disease progression following standard CXL procedure amounts to $2.9-7.6 \%$ and depends on the stage of keratoconus [17]. Progression of the disease despite CXL procedure has been proven to occur more frequently in patients with baseline $\mathrm{K}_{\max }$ values above $58 \mathrm{D}[17,18]$.

\section{Functional Results and Morphometric Parameters of Cornea Following the Standard Cxl Procedure}

The conclusions of majority of studies analysing outcome of standard CXL procedure indicate some improvement in visual acuity in keratoconus patients [5]. A significantly lower number of papers report lack of such effect [19-21]. CXL procedure is known to reduce both $\mathrm{K}_{\max }$ and $\mathrm{K}_{\min }$ values [22,23]. Such changes are usually noted during the first year after the procedure. However, it should be mentioned that there are cases of delayed change of $\mathrm{K}_{\max }$ value, occurring as late as between the $24^{\text {th }}$ and $36^{\text {th }}$ month of the followup [22-24]. Significant corneal flattening $\left(\Delta K_{\max }>1\right.$ D) occurs in over $50 \%$ patients within one year after the procedure, while in $13 \%$ of them the change amounts to even $>2$ D [25]. Some studies suggest that corneal flattening following CXL is correlated with the stage of disease progressions. Some authors noted more marked corneal flattening in more advances keratoconus cases, with $\mathrm{K}_{\max }$ values within 54-58 D range[22,25].Conversely, other authors reported more significant corneal flattening in initial stages of keratoconus, with $\mathrm{K}_{\max }$ values $\leq 53 \mathrm{D}[26]$.

According to some studies, changes of corneal thickness are observed after CXL procedure. Independent analyses by Witting-Sliva et al. and Caprossi et al. indicated that there were no differences in corneal thickness as assessed by ultrasound pachymetry (USP) during 3-year follow-up; however, examinations performed by means of Orbscan apparatus revealed significant corneal thinning within first 3 month after the procedure [22]. Results of corneal thickness measurements performed by means of Orbscan apparatus, HRT, confocal pachymetry and OCT were equivocal [27]. Other authors demonstrated significant differences in corneal thickness after standard CXL procedure in examinations with Scheimpflug camera at various stages of follow-up Topraket et al. [28] and Greenstein et al. [29] reported reduction in corneal thickness in 6th and 12th month, respectively. Hashemi et al. noted significant corneal thinning in 1st month after standard CXL procedure. The authors also stress the fact that in subsequent check-ups corneal thickness increased gradually up to 6th month after the procedure, achieving values similar to baseline ones [19].

The general opinion is that corneal thickness changes after the CXL procedure results from the keratocyte restoration process that lasts approx. 6 months. Additionally, the rearrangement of the corneal lamellae, anatomic and structural changes of the collagen fibres and changes in corneal stroma (glycosaminoglycans) last approx. 2 years after the procedure [30]. Other authors explain the changes with the damage and subsequent restoration of nerve fibres, which may affect the corneal sensation and endothelial pump performance [31]. Corneal ischaemia, a tighter arrangement of collagen lamellae and changes in the arrangement of the restored corneal epithelium are listed among the other possible causes [10].

\section{Modifications of cross-linking procedure}

Attempts to reduction the cross-linking procedure duration are based on shorter riboflavin administration time (e.g. with the use of iontophoresis) or on the application of higher UV doses (accelerated cross-linking, fast cross-linking, rapid cross-linking). Theoretical background for the modification of the method is based on the photochemical reciprocity law (Bunsen-Roscoe law), according to which rate of the photochemical and photobiological reaction is directly proportional to the total dose of radiation energy. It means in general, that similar effect of corneal cross-linking, similar to that achieved with the standard CXL method, may be achieved with various intensity and duration of UVA irradiation, provided that an identical total dose of energy is delivered[32,33].

Experimental trials have demonstrated that prolongation of exposure to light up to 60 minutes, and thus exceeding UVA energy dose above $5.4 \mathrm{~J} / \mathrm{cm}^{2}$, does not result in the outcome similar to this of standard method, while biomechanical strength of such cornea is similar to cornea not exposed to CXL irradiation procedure [34]. Moreover, exceeding the safe radiation dose, considered to be 5.4 $\mathrm{J} / \mathrm{cm}^{2}$ may significantly increase the risk of phototoxic intraocular complications [2,5]. Thus, modifications of cross-linking procedure consist in the proportional change of UVA irradiation duration and intensity, with a constant safe dose of total radiation dose energy. The majority of studies evaluating the efficacy and safety of modifications of alternative CXL methods involve experimental studies on the porcine cornea (Figure 3).

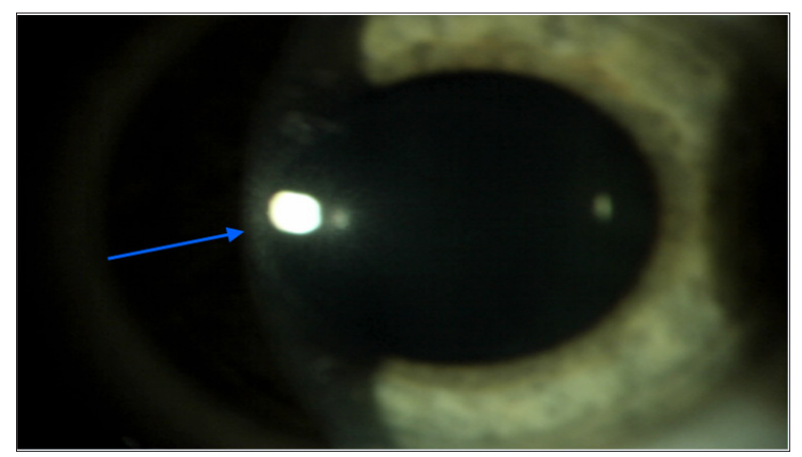

Figure 3: Corneal haze after CXL procedure (blue arrow).

There are only several reports on the outcome of accelerated cross-linking in keratoconus patients available in medical 
literature. Schumacher et al. demonstrated the equal efficacy of the accelerated cross-linking and standard CXL procedure on the porcine cornea, with UVA irradiation at a power of $10 \mathrm{~mW} / \mathrm{cm}^{2}$ for 9 minutes [33]. Wernli et al. [35] noted an increased corneal stiffness in line with the increase of UVA intensity to $45 \mathrm{~mW} / \mathrm{cm}^{2}$ and a decrease in the exposure time to 2 minutes. On the other hand, with combination of higher UVA intensity (above $50 \mathrm{~mW} / \mathrm{cm}^{2}$ ) and shorter irradiation duration, no significant changes of corneal stiffness was demonstrated. Interestingly, the most recent reports stress the fact that higher UVA intensity and shorter irradiation time result in poorer CXL outcome [36]. The effect is explained by increased oxygen consumption and, consequently, lower efficiency of photochemical reactions during fats CXL procedure [37].This is supported by results indicating the complete lack of effects of the CXL performed in anaerobic conditions [38].

Postoperative results of accelerated CXL procedure are equivocal. There are literature reports on significant reduction of $\mathrm{K}_{\max }$ value and improvement in visual acuity, as well as papers indicating no significant changes in keratometry results [39-40]. Reduced keratocyte destruction would be a strong argument supporting the development of alternative CXL methods [39]. However, such a claim was not confirmed by results of the corneal confocal microscopy in patients following the fast CXL procedure (in modification involving $30 \mathrm{~mW} / \mathrm{cm}^{2}$ UVA for 3 minutes). The results revealed more intense morphological changes than following a standard CXL procedure, as well as increased keratocytes apoptosis, tissue hyper reflectivity, and more numerous and darker stromal defects [41].

Another CXL modification, enabling protection of corneal epithelial, is "Epithelium-On" method.1 The following methods are applied to increase vitamin $B_{2}$ penetration through intact corneal epithelium: viscous riboflavin solution, the use of a ring limiting outflow of the solution, modification of its osmolarity or riboflavin concentration and/or the use of iontophoresis. Better penetration may be also achieved thanks to increased epithelial permeability due to loosening of intercellular junctions following administration of benzalkonium chloride (BAC), ethylenediaminetetraacetic acid (EDTA) or NC-1059 peptide, or thanks to formation of pockets for riboflavin administration within corneal stroma, linear, grid-like incisions of corneal epithelium or removal of superficial epithelial layer by means of excimer laser [32].

To date, numerous critical data concerning this method were published, indicating poorer control of keratoconus progression, as compared to standard CXL method [42]. It has been suggested to result from reduced penetration of riboflavin through the layers of epithelium and Bowman's membrane, as well as uneven distribution of riboflavin within corneal stroma. Moreover, presence of dextran in riboflavin solution has been proven to additionally reduce penetration of riboflavin through epithelium [43].Also epithelial cells reduce penetration of UVA by approx. $20-30 \%$.

Some reports stress that total dose of UVA energy applied in Epithelium-On should be $7.2 \mathrm{~J} / \mathrm{cm}^{2}$ to be equivalent to standard CXL procedure. The claim is supported by studies by E. Yuksel et al. $[44,45]$, that involved over a 1 year follow- up of keratoconus progression inhibition after Epithelium-On CXL procedure involving the use of suggested dextran-free riboflavin dose and UVA power. Despite multiple modifications and attempts to combine CXL methods with refractive surgery of cornea and lens, standard CXL is currently the most common method of keratoconus therapy. However, problems and complications inherent to the procedure methodology are still a strong argument for seeking alternative methods.We wish to confirm that there are no conflicts of interest associated with this publication and there has been no financial support for this work that could influence its outcome.

\section{References}

1. Raiskup F, Spoerl E (2013) Corneal cross linking with riboflavin and ultraviolet A Part II Clinical indications and results. Ocul Surf 11: 93-108.

2. Spoerl E, Mrochen M, Sliney D, Trokel S, Seiler T, et al. (2007) Safety of UVA-riboflavin cross-linking of the cornea. Cornea 26: 385-389.

3. Wollensak G, Spörl E, Reber F, Pillunat L, Funk R, et al. (2003) Corneal endothelial cytotoxicity of riboflavin/UVA treatment in vitro. Ophthalmic Res 35: 324-328.

4. Hafezi F, Randleman JB (2014) PACK-CXL: defining CXL for infectious keratitis. J Refract Surg 30: 438-439.

5. Wollensak G (2010) Corneal collagen crosslinking: new horizons. Expert Rev Ophthalmol 5: 201-215.

6. Choy BN, Zhu MM, Shum JW, Ho WL, Chan JC, et al. (2016) Collagen crosslinking in the management of leaking cystic blebs: a prospective study. Graefes Arch ClinExpOphthalmo 254: 529-533.

7. Dotan A, Kremer I, Gal Or O, Livnat T, Zigler A, et al. (2016) Ultraviolet-A radiation for prevention of axial myopia in a rabbit model. J Vis Exp.

8. Ghanem VC, Ghanem RC, de Oliveira R (2013) Postoperative pain after corneal collagen cross-linking. Cornea 32: 20-24.

9. Mastropasqua L (2015) Collagen cross-linking: when and how? A review of the state of the art of the technique and new perspectives. Eye and Vision 2: 19.

10. Holopainen JM, Krootila K (2011) Transient corneal thinning in eyes undergoing corneal cross-linking. Am J Ophthalmol 152: 533-536.

11. Tahzib NG, Soetern N, Van der Lelij A (2010) Pachymetry during crosslinking. Ophthalmology 117: 2041.

12. Schmidinger G, Pachala M, Prager F (2013) Pachymetry changes during corneal crosslinking: effect of closed eyelids and hypotonic riboflavin solution. J Cataract Refract Surg 39: 1179-1183.

13. Kymonis GD, Grentzelos MA, Stojanovic N, Paraskevopoulos TA, et al. (2014) Transient an socoria after corneal collagen cross-linking. Case Rep Ophthalmol Med 2014: 487860.

14. Yuksel N, Bilgihan K, Hondur AM (2011) Herpetic keratitis after corneal collagen cross-linking with riboflavin and ultraviolet-A for progressive keratoconus. Int Ophthalmol 31(6): 513-515.

15. Al Amry M, Mudhaiyan T, Al Huthail R, Al Ghadeer H (2017) Infectious Crystalline Keratopathy After Corneal Cross-linking. Middle East Afr J Ophthalmol 24: 100-102.

16. Gumus K (2014) Acute idiopathic endo the liit is early efter corneal crosslinking with riboflavin and ultraviolet A Cornea 33: 630-633.

17. Koller T, Mrochen M, Seiler T (2009) Complication and failure rates after corneal crosslinking. J Cataract Refract Surg 35: 1358-1362.

18. Mazzotta C, Balestrazzi A, Traversi C, Baiocchi S, Caporossi T, et al. (2007) Treatment of progressive keratoconus by riboflavin-UVA-induced crosslinking of corneal collagen; ultrastructural analysis by Heidelberg 
Retinal Tomograph II in vivo confocal microscopy in humans. Cornea 26(4): 390-397.

19. Hashemi H, Seyedian MA, Miraftab M, Fotouchi A, Asgari S, et al. (2013) Corneal collagen cross-linking with riboflavin and ultraviolet a irradiation for keratoconus: long-term results. Ophthalmology 120: 1515-1520.

20. Asri D, Touboul D, Fournie P, Malet F, Garra C, et al. (2011) Corneal collagen crosslinking in progressive keratoconus: multicenter results from the French National Reference Center for Keratoconus. J Cataract Refract Surg 37: 2137- 2143

21. Grewal DS, Brar GS, Jain R, Sood V, Singla V, et al. (2009) Corneal collagen cross linking using riboflavin and ultraviolet- A light for keratoconus: one-year analysis using Scheimpflug imaging. J Cataract Refract Surg 35(3):425-432.

22. Wittig Silva C, Chan E, Islam FM, Wu T, Whiting M, et al. (2014) A randomized, controlled trial of corneal collagen cross-linking in progressive keratoconus: three-year results. Ophthalmology 121: 812821.

23. Vinciguerra P, Albè E, Frueh BE, Trazza S, Epstein D (2012) Two-Year Corneal Cross-Linking Results in Patients Younger Than 18 Years With Documented Progressive Keratoconus. Am J Ophthalmol 154: 520-526.

24. O’Brart DP, Kwong TQ, Patel P, McDonald RJ, O Brart NA, et al. (2013) Long-term follow-up of riboflavin/ultraviolet A $(370 \mathrm{~nm})$ corneal collagen crosslinking to halt the progression of keratoconus. Br J Ophthalmol 97: 433-437.

25. Koller T, Pajic B, Vinciguerra P, Seiler T (2011) Flattening of the cornea after collagen cross linking for keratoconus. J Cataract Refract Surg. 37: 1488-1492.

26. Arora R, Jain P, Goyal JL, Gupta D (2013) Comparative Analysis of Refractive and Topographic Changes in Early and Advanced Keratoconic Eyes Undergoing Corneal Collagen Crosslinking. Cornea 22: 1359-1364.

27. Caporossi A, Mazzotta C, Baiocchi S, Caporossi T (2010) Long-term results of riboflavin ultraviolet a corneal collagen cross-linking for keratoconus in Italy: the Siena eye cross study. Am J Ophthalmol 149: 585-593.

28. Toprak I, Yildirim C (2013) Scheimpflug parameters after corneal collagen crosslinking for keratoconus. Eur J Ophthalmol 23: 793-798.

29. Greenstein SA, Shah VP, Fry KL, Hersh PS (2011) Corneal thickness changes after corneal collagen crosslinking for keratoconus and corneal ectasia: one-year results. J Cataract Refract Surg 37: 691-700.

30. MazzottaC, Traversi C, Baiocchi S, Caporossi O, Bovone C, et al. (2008) Corneal healing after riboflavin ultraviolet-A collagen cross-linking determined by confocal laser scanning microscopy in vivo: early and late modifications. Am J Ophthalmol 146: 527-533.

31. Arita R, Arita M, Kawai M, Mashima Y, Yamada M (2005) Evaluation of corneal endothelial pump function with a cold stress test. Cornea 24: 571-575.

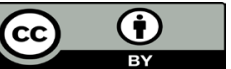

This work is licensed under Creative Commons Attribution 4.0 License

Submission Link: http://biomedres.us/submit-manuscript.php
32. Raiskup F, Spoerl E (2013) Corneal cross linking with riboflavin and ultraviolet A I Principles. Ocul Surf 11: 65-74.

33. Schumacher S, Oeftiger L, Mrochen M (2011) Equivalence of biomechanical changes induced by rapid and standard corneal crosslinking, using riboflavin and ultraviolet radiation. Invest Ophthalmol Vis Sci 52: 9048-9052.

34. Lanchares E, del Buey MA, Cristóbal JA, Lavilla L, Calvo B, et al. (2011) Biomechanical property analysis after corneal collagen cross-linking in relation to ultraviolet A irradiation time. Graefes Arch ClinExpOphthalmol 249: 1223-1227.

35. Wernli J, Schumacher S, Spoerl E, Mrochen M (2013) The efficacy of corneal cross-linking shows a sudden decrease with very high intensity UV light and short treatment time. Invest Ophthalmol Vis Sci 54: 1176-1180.

36. Hammer A, Richoz O, Mosquera S, Tabibian D, Hoogewoud F, et al. (2014) Corneal biomechanical properties at different corneal collagen crosslinking (CXL) Irradiances. Invest Ophthalmol Vis Sci 55: 2881-2884.

37. Mrochen M (2013) Current status of accelerated corneal cross linking. Indian J Ophthalmol 61: 428-429.

38. Kamaev P, Friedman MD, Sherr E, Muller D (2012) Photochemical kinetics of corneal cross-linking with riboflavin. Invest Ophthalmol Vis Sci 53: 2360-2367.

39. Kanellopoulos AJ (2012) Long term results of a prospective randomized bilateral eye comparison trial of higher fluence, shorter duration ultraviolet A radiation, and riboflavin collagen cross linking for progressive keratoconus. ClinOphthalmol 6: 97-101.

40. Waszczykowska A, Jurowski P (2015) Two-year accelerated corneal cross-linking outcome in patients with progressive keratoconus. Biomed Res Int: pp. 325157.

41. Touboul D, Efron N, Smadja D, Praud D, Malet F, et al. (2012) Corneal confocal microscopy following conventional, transepithelial, and accelerated corneal collagen cross-linking procedures for keratoconus. J Refract Surg 28: 769-776.

42. Caporossi A, Mazzotta C, Paradiso AL, Baiocchi S, Marigliani D, et al. (2013) Transepithelial corneal collagen crosslinking for progressive keratoconus: 24-month clinical results. J Cataract Refract Surg 39: 11571163.

43. Bottos KM, Schor P, Dreyfuss JL, Nader HB, Chamon W, et al. (2011) Effect of cornealepithelium on ultraviolet-A and riboflavinabsorption. Arq Bras Oftalmol 74: 348-351.

44. Yuksel E, Cubuk M, Yalcin G, Ozmen M, Aydin B, et al. (2015) Epithelial on or epithelial off corneal collagen cross-linking:bilateral comparison study. 19th ESCRS Winter Meeting, Istanbul, Turkey; p. 20-22.

45. Yuksel E, Bektas C, Bilgihan K (2015) Transepithelial Versus Epitheliumoff Corneal Cross-Linking for the Treatment of Progressive Keratoconus: A Randomized Controlled Trial. Am J Ophthalmol. 160(2): 399-400.

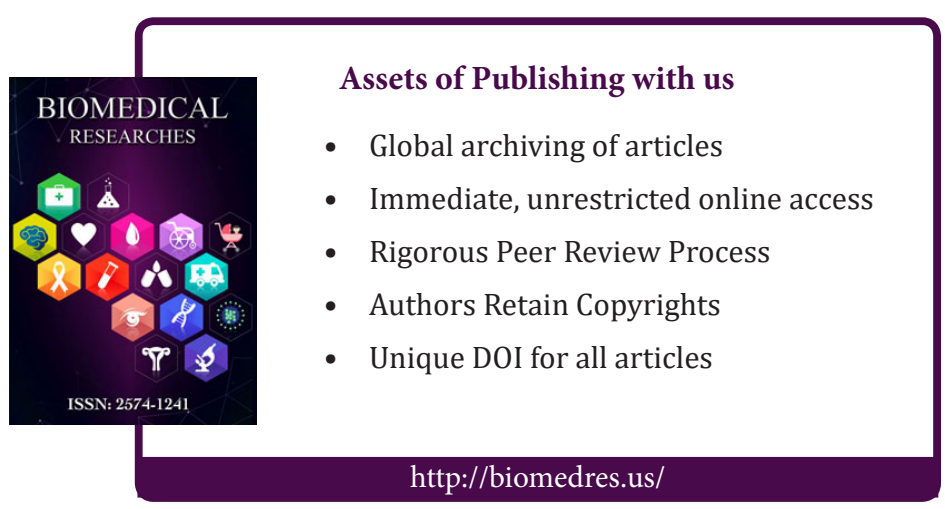

